\title{
Terapêutica da Enxaqueca: Avanços Recentes
}

\section{Migraine Therapy: Recent Advances}

José M. Pereira Monteiro ${ }^{1,2,3}$

\section{RESUMO}

Neste artigo são sintetizados os conceitos atuais sobre a fisiopatogenia e as manifestações clínicas da enxaqueca, assim como as terapêuticas disponíveis com destaque para as novas terapêuticas preventivas e suas implicações clínicas e sociais.

PALAVRAS-CHAVE: Antagonistas do Recetor do Peptídeo Relacionado ao Gene de Calcitonina; Enxaqueca/fisiopatologia; Enxaqueca/tratamento; Peptídeo Relacionado ao Gene de Calcitonina

\section{ABSTRACT}

This paper summarizes the most recent concepts on the pathophysiology and clinical features of migraine as well as the available therapies with special focus on the new specific preventive therapies and its clinical and social effects.

KEYWORDS: Calcitonin Gene-Related Peptide; Calcitonin Gene-Related Peptide Receptor Antagonists; Migraine Disorders/ drug therapy; Migraine Disorders/physiopathology 


\section{INTRODUÇÃO}

O objetivo da revisão foi apresentar os conceitos atuais sobre a fisiopatogenia e clínica da enxaqueca e sobre as novas terapêuticas preventivas específicas e suas implicações clínicas e sociais.

\section{ENXAQUECA}

A enxaqueca é uma entidade clínica caraterizada por acessos (crises) de dores de cabeça uni ou bilaterais de início insidioso mas progressivo, atingindo uma intensidade moderada a severa, geralmente de caráter pulsátil e acompanhados de intolerância à luz (fotofobia), aos ruídos (fonofobia), aos cheiros (osmofobia) e/ou aos movimentos da cabeça ou do corpo (cinesifobia) e de náuseas e/ou vómitos na maior parte das crises. As crises, contudo, iniciam-se, frequentemente, cerca de 48 horas antes do início da dor, por sintomas mal definidos como irritabilidade, dificuldade de concentração mental, cansaço e bocejo. A duração total da crise varia de 4 a 72 horas, podendo ser menor em algumas crises e nas crianças (2 horas) e mais duradoira nas formas crónicas e com uso excessivo de medicação analgésica. ${ }^{1}$ Ocorre com uma frequência variável desde menos de uma crise por mês, nas formas episódicas pouco frequentes, até uma por dia nas formas crónicas e/ou com uso excessivo de medicação abortiva das crises. ${ }^{1}$

Tem uma prevalência muito elevada na população geral, ${ }^{2-6}$ sendo a prevalência anual mundial de 15\% - 18\%, ${ }^{4}$ embora com variações geográficas ${ }^{3}$ sendo de 16,6\% em Portugal ${ }^{7}$ e com claro predomínio no género feminino (3:1).4-7 Inicia-se, geralmente, na segunda década da vida e tem um pico de incidência entre a segunda e a quarta década da vida, isto é, na fase mais produtiva da vida. Por isso foi considerada pela Organização Mundial de Saúde (OMS) como a sexta doença mais prevalente a nível mundial afetando mais de 1 bilião de pessoas ${ }^{3,5,8}$ sendo a doença neurológica mais prevalente. ${ }^{3,5,6,8}$

Pode ocorrer com distintas formas de apresentação clínica conforme contenha sintomas ou sinais neurológicos focais transitórios iniciais a que chamamos de aura designando-se por enxaqueca com aura, a qual pode ser visual, sensitiva ou motora ou combinações destas, sendo a sua frequência variável entre as diferentes séries $(5 \%-10 \%)^{6,9}$ ou sem esses sintomas e designando-se por enxaqueca sem aura, sendo esta a forma mais frequente (> 60\%), ,7,9-11 podendo ocorrer, no mesmo indivíduo, ambas as formas de apresentação clínica em diferentes crises (30\% - 35\%). 5,6,8

As crises acompanham-se de graus variáveis de incapacidade funcional profissional ou escolar podendo chegar a ser totalmente incompatíveis com qualquer atividade durante toda ou parte da duração da crise e a sua frequência, se elevada, perturba muito o desempenho individual, interfere significativamente na qualidade de vida dos indivíduos afetados, tem implicações económicas (diretas e indiretas) notórias além de repercussões pessoais, familiares e sociais relevantes. . $-6,8-12^{-12}$

O reconhecimento desta incapacidade funcional pela OMS em 2017, ao atribuir-Ihe a segunda causa de anos vividos com incapacidade sendo a primeira causa de incapacidade entre os anos de 18 e 50 anos de idade a nível mundial ${ }^{10}$ contribuiu decisivamente para o crescente reconhecimento mundial desta patologia, embora ainda esteja muito aquém do que a doença requer.

A etiologia permanece desconhecida apesar dos muitos esforços feitos por muitos investigadores para tentar identificar a sua causa. ${ }^{13,14} \mathrm{~A}$ incidência familiar é muito evidente $(>60 \%)^{6,7,11}$ sugerindo uma origem genética, tendo sido identificada numa variante com aura motora (enxaqueca hemiplégica familiar) as seguintes mutações genéticas CACNA1A, ATP1A2 e SCNA1 associadas e que são designadas por tipos 1, 2 e 3.15-17 Contudo, ainda não foi possível identificar uma mutação patogénica na enxaqueca com e sem aura ${ }^{14,18}$ e apenas diversos polimorfismos associados foram identificados ${ }^{18-20}$ admitindo-se para já que se trata de uma afeção de hereditariedade complexa e multifatorial.

Atualmente, considera-se que a sua origem é primariamente neuronal e no sistema nervoso central (córtex cerebral), ${ }^{21,22}$ possivelmente resultante de uma perturbação do metabolismo energético mitocondrial, e com repercussão secundária subcortical, neurovascular e sistémica. O início das crises tem sido atribuído ao desenvolvimento de uma depressão cortical ${ }^{22}$ começando no córtex occipital e alastrando para as estruturas adjacentes: parietal, temporal e frontal (depressão cortical alastrante) e estruturas subcorticais com envolvimento do tronco cerebral, do núcleo trigeminal e através dele, ativando o sistema trigémino-vascular causando vasodilatação meníngea e a libertação de substâncias algógenas para o espaço perivascular (inflamação neurogénea estéril) e a subsequente dor pulsátil..21-24

Estudos laboratoriais identificaram um défice de serotonina ${ }^{25}$ pelo que foi advogado o uso de agonistas serotoninérgicos como potenciais fármacos indicados para o tratamento da fase aguda da enxaqueca. Assim surgiram os triptanos (sumatriptano, zolmitriptano, naratriptano, almotriptano, rizatriptano, frovatriptano e eletriptano) que são os fármacos mais eficazes no tratamento da crise de enxaqueca. ${ }^{26}$ 
Estudos laboratoriais mais recentes permitiram identificar a ativação da via trigémino-vascular e a libertação do CGRP, um potente vasodilatador, durante as crises. ${ }^{27-29}$ Verificou-se que os triptanos, fármacos usados no tratamento das crises de enxaqueca, reduzem o CGRP. ${ }^{29}$ Esta substância tornou-se, nos últimos anos, uma espécie de marcador da enxaqueca e um alvo apetecido para o tratamento desta entidade clínica.

Foram então desenvolvidos fármacos antagonistas do CGRP, de administração oral, designados genericamente por "gepants" mas, os seus efeitos secundários, nomeadamente hepatotoxicidade, impediram o seu desenvolvimento pelo que, posteriormente, foram desenvolvidos anticorpos monoclonais contra o CGRP ou os seus recetores ${ }^{30-33}$ que ultrapassaram eficazmente essas limitações, mas continuam em desenvolvimento novos "gepants" que não têm os efeitos hepatotóxicos dos primeiros e também estão a ser desenvolvidos anticorpos monoclonais contra o CGRP para administração oral o que faz prever um crescimento exponencial das disponibilidades terapêuticas para a enxaqueca num futuro próximo.

Atualmente há três anticorpos monoclonais anti-CGRP, todos de administração parentérica, nomeadamente: fremanezumab (SC-trimestral ou mensal), eptinezumab (EV-mensal) e o galcanezumab (SC-mensal) e um anticorpo antirecetor do CGRP o erenumab (SC-mensal), este já comercializado em Portugal. $33-35$

Estes anticorpos monoclonais têm-se revelado, em estudos controlados contra placebo, eficazes na prevenção das crises de enxaqueca com e sem aura, episódica e crónica, mesmo quando há uso excessivo de analgésicos ou se apresenta como refratária aos tratamentos agudos ou preventivos tradicionais, verificando-se uma redução sustentada dos dias de cefaleias por mês, da necessidade de consumo de analgésicos com melhoria da incapacidade associada à enxaqueca e da qualidade de vida sendo muito bem tolerados pelo que poderão vir a revolucionar a terapêutica da enxaqueca especialmente se o seu custo os tornar acessíveis para uso generalizado na população afetada. ${ }^{36}$

\section{CONCLUSÃO}

Sendo a enxaqueca uma afeção altamente prevalente e temporariamente incapacitante, com enorme repercussão na qualidade de vida dos indivíduos afetados, apesar de ser muito desvalorizada em termos clínicos e sociais, ganhou o merecido destaque com o reconhecimento mundial pela OMS da incapacidade funcional que the está associada e o interesse da indústria farmacêutica para o desenvolvimento de fármacos específicos, quer para o tratamento da fase aguda (crise) com os triptanos desenvolvidos na década de 90 do século passado, quer para o tratamento preventivo com os anticorpos monoclonais dirigidos ao CGRP ou aos seus recetores, desenvolvidos na última década e que continuam em desenvolvimento que, pela sua eficácia e segurança, poderão vir a revolucionar a terapêutica da enxaqueca especialmente se o seu custo os tornar acessíveis a toda a população afetada constituindo assim, no seu conjunto um armamentário terapêutico permitindo controlar eficazmente a sintomatologia associada às crises de enxaqueca assim como prevenir a sua ocorrência e contribuir para redução substancial da incapacidade associada a esta doença e melhorar substancialmente a qualidade de vida destes doentes bem como reduzir significativamente os riscos associados ao uso excessivo de medicação analgésica, incluindo narcóticos e sedativos, com os inerentes riscos associados de habituação e dependência constituindo uma renovada esperança para os portadores da enxaqueca e seus familiares.

CONFLITOS DE INTERESSE: O autor é Consultor da Novartis-Farma.

FONTES DE FINANCIAMENTO: Este artigo insere-se na atividade do projeto de investigação PTDC/MEC-NEU/29486/2017 da FCT.

PROVENIÊNCIA E REVISÃO POR PARES: Não comissionado; revisão externa por pares.

CONFLICTS OF INTEREST: The author is Novartis-Farma Consultant.

FINANCIAL SUPPORT: This article is part of the FCT research project activity PTDC/MEC-NEU/29486/2017.

PROVENANCE AND PEER REVIEW: Not commissioned; externally peer reviewed.

\section{REFERÊNCIAS}

1. Headache Classification Committee of the International Headache Society (IHS). The International Classification of Headache Disorders, 3rd edition. Cephalalgia. 2018; 38: 1-211.

2. Wolde Manuel YW, Cowan RP. Migraine affects 1 in 10 people worldwide featuring recent rise: A systematic review and metanalysis of community-based studies involving 6 million participants. J Neurol Sci. 2017; 372: 307-15. doi: 10.1016/j. jns.2016.11.071.

3. GBD 2016 Disease and Injury Incidence and Prevalence Collaborators. Global, regional, and national incidence, prevalence, and years lived with disability for 328 diseases and injuries for 195 countries, 1990-2016: a systematic analysis for the Global Burden of Disease Study 2016. Lancet. 2017;390:121159. doi: 10.1016/S0140-6736(17)32154-2.

4. Stovner LJ, Hagen K, Jensen R, Katsarava Z, Lipton RB, Scher $\mathrm{Al}$, et al. The global burden of headache: a documentation of headache prevalence and disability worldwide. Cephalalgia. 2007; 27:193-210. 
5. Global Burden of Disease Study. Global, regional, and national incidence, prevalence, and years lived with disability for 301 acute and chronic diseases and injuries in 188 countries, 1990-2013: a systematic analysis for the Global Burden of Disease Study 2013. Lancet. 2015; 386: 743-800. doi: 10.1016/ S0140-6736(15)60692-4.

6. Stovner LJ, Andree C. Prevalence of headache in Europe: a review for the Eurolight project. J Headache Pain. 2010; 11:289-99. doi: 10.1007/s10194-010-0217-0.

7. Pereira Monteiro JM. Cefaleias: estudo epidemiológico e clínico de uma população urbana. Porto: Universidade do Porto; 1995.

8. Lipton RB, Bigal ME, Diamond M, Freitag F, Reed ML, Stewart WF; AMPP Advisory Group. Migraine prevalence, disease burden, and the need for preventive therapy. Neurology. 2007; 68:343-9.

9. Stewart WF, Ricci JA, Chee E, Morganstein D, Lipton R. Lost productive time and cost due to common pain conditions in the US workforce. JAMA. 2003; 290: 2443-2454.

10. Steiner T, Stovner J, Vos T, Jensen R, Katsarava K. Migraine is the first cause of disability under 50s: will health politicians now take notice? J Headache Pain. 2018; 19: 17. doi: 10.1186/ s10194-018-0846-2.

11. Rasmussen BK, Olesen J. Migraine with aura and migraine without aura: an epidemiological study. Cephalalgia. 1992; 12: 221-8.

12. Giffin NJ, Ruggiero L, Lipton RB, Silberstein S, Tvedskov JF, Olesen J, et al. Premonitory symptoms in migraine: an electronic diary study. Neurology. 2003; 60: 935-40.

13. Bashir A, Lipton RB, Ashina S, Ashina M. Migraine and structural changes in the brain: a systematic review and meta-analysis. Neurology.2013;81:1260-8. doi: 10.1212/ WNL.Ob013e3182a6cb32.

14. Tolner EA, Houben T, Terwindt GM, de Vries B, Ferrari MD, van den Maagdenberg AM. From migraine genes to mechanisms. Pain. 2015;156: -S64-74.

15. Ophoff RA, Terwindt GM, Vergouwe MN, van Eijk R, Oefner PJ, Hoffman SM, et al. Familial hemiplegic migraine and episodic ataxia type- 2 are caused by mutations in the $\mathrm{Ca} 2 \mathrm{p}$ channel gene CACNL1A4. Cell. 1996; 87:543-52.

16. De Fusco M, Marconi R, Silverstri L, Atorino L, Rampoldi L, Morgante L, et al. HaploinsuYciency of ATP1A2 encoding the $\mathrm{Na}+/ \mathrm{K}+$ pump alpha2 subunit associated with familial hemiplegic migraine type 2. Nat Genet. 2003; 33:192-6.

17. Dichgans M, Freilinger T, Eckstein G, Babini E, Lorenz-Depiereux B, Biskup S, et al. Mutation in the neuronal voltage-gated sodium channel SCN1A in familial hemiplegic migraine. Lancet. 2005;336:371-7.

18. Chasman DI, Schurks M, Anttila V, de Vries B, Schminke U, Launer $L J$, et al.Genome-wide association study reveals threes usceptibility loci for common migraine in the general population. Nat Genet. 2011;43: 695-8. doi: 10.1038/ng.856.

19. Freilinger T, Anttila V, de Vries B, Malik R, Kallela M, Terwindt GM, et al. Genome-wide association analysis identifies susceptibility loci for migraine without aura. Nat Genet. 2012:44:777-8. doi: 10.1038/ng.2307.

20. Chasman DI, Schurks M, Anttila V, de Vries B, Schminke U, Launer LJ, et al. Genome-wide association study reveals three susceptibility loci for common migraine in the general population. Nat Genet.2011;43:695-8. doi: 10.1038/ng.856.

21. Charles A. Migraine: a brain state. Curr Opin Neurol. 2013;26:235-9. doi: 10.1097/WCO.0b013e32836085f4.

22. DiMauro S, Schon EA. Mitochondrial respiratory-chain diseases. N Engl J Med. 2004;348:2556-68.
23. Weiller C, May A, Limmroth V, Juptner M, Kaube H, Schayck $\mathrm{RV}$, et al. Brain stem activation in spontaneous human migraine attacks. Nature Med. 1995;1:658-60.

24. Hansen JM, Baca SM, Van Valkenburgh P, Charles A. Distinctive anatomical and physiological features of migraine aura revealed by 18 years of recording. Brain. 2013;136:3589-95.

25. Curran DA, Hinterberger H, Lance JW. Total plasma serotonin, 5-hydroxyindoleacetic acid and p-hydroxy-m-methoxymandelic acid excretion in normal and migrainous subjects. Brain. 1965;88:997-1010.

26. Classey JD, Bartsch T, Goadsby PJ. Distribution of 5-HT1B, 5-HT1D and 5-HT1F receptor expression in rat trigeminal and dorsal root ganglia neurons: relevance to the selective anti-migraine effect of triptans. Brain Res. 2010;1361:76-85. doi: 10.1016/j.brainres.2010.09.004.

27. Goadsby PJ, Edvinsson L, Ekman R. Vasoactive peptide release in the extracerebral circulation of humans during migraine headache. Ann Neurol. 1990;28:183-7.

28. Summ O, Charbit AR, Andreou AP, Goadsby PJ. Modulation of nocioceptive transmission with calcitonin gene-related peptide receptor antagonists in the thalamus. Brain. 2010;133:2540-8. doi: 10.1093/brain/awq224.

29. Ho TW, Edvinsson L, Goadsby PJ. CGRP and its receptors provide new insights into migraine pathophysiology. Nature Rev Neurol. 2010;6:573-82. doi: 10.1038/nrneurol.2010.127.

30. Doods H, Hallermayer G, Wu D, Entzeroth M, Rudolf K, Engel W, et al. Pharmacological profile of BIBN4096BS, the first selective small molecule CGRP antagonist. $\mathrm{Br} J$ Pharmacol. 2000;129:420-3.

31. Bigal ME, Dodick DW, Rapoport AM, Silberstein SD, Ma Y, Yang R, et al. Safety, tolerability, and efficacy of TEV-48125 for preventive treatment of high-frequency episodic migraine: a multicentre, randomised, double-blind, placebo-controlled, phase 2b study. Lancet Neurol. 2015;14: 1081- 90.

32. Bigal ME, Escandon R, Bronson M, Walter S, Sudworth M, Huggins JP, et al. Safety and tolerability of LBR-101, a humanized monoclonal antibody that blocks the binding of CGRP to its receptor: results of the Phase 1 program. Cephalalgia. 2013;34: 483-92. doi: 10.1177/0333102413517775.

33. Sun H, Dodick DW, Silberstein S, Goadsby PJ, Reuter U, Ashina $\mathrm{M}$, et al. A randomised, double-blind, placebo-controlled, phase2 study to evaluate the efficacy and safety of AMG 334 for the prevention of episodic migraine. Lancet Neurol. 2016;15: 382-90.

34. Bigal ME, Escandon R, Bronson M, Walter S, Sudworth M, Huggins JP, et al. Safety and tolerability of LBR-101, a humanized monoclonal antibody that blocks the binding of CGRP to its receptor: results of the Phase 1 program. Cephalalgia. 2013;34:483-92. doi: 10.1177/0333102413517775.

35. Cernuda-Morollon E, Larrosa D, Moris G, Ramon C, Pascual J. Increased levels of CGRP in peripheral blood in women with chronic migraine: A reliable biological marker. J Headache Pain. 2013; 14: P104.

36. Silberstein S, Holland S, Freitag F, Dodick DW, Argoff C, Ashman E, et al. Evidence-based guideline update: Pharmacologic treatment for episodic migraine prevention in adults: Report of the quality standards subcommittee of the American Academy of Neurology and the American Headache Society. Neurology. 2012; 78; 1337-45. 